\title{
A Computer Navigation System Analysis of the Accuracy of the Extramedullary (Tibial) Alignment Technique in Total Knee Arthroplasty (TKA)
}

\author{
EK Chee, MS Ortho, TW Ewe, FRCS \\ Department of Orthopaedics and Traumatology, Hospital Kuala Lumpur, Kuala Lumpur, Malaysia
}

\begin{abstract}
In total knee arthroplasty, mechanical alignment guides have improved the accuracy of implant alignment, but errors are not uncommon. In the present study, an image-free computer-assisted navigation system was used to analyse the accuracy of an extramedullary (tibial) alignment system, which is based on predetermined, fixed anatomical landmarks. Comparisons were made between two surgeons, with different levels of competency in order to determine if experience affected the accuracy of extramedullary tibial jig placement, in either the coronal and sagittal planes or both planes. The results showed that the accuracy of the extramedullary tibial alignment system, in the coronal plane (in up to $80-87 \%$ of cases) was much better than for posterior slope, and sagittal plane. Surgeon experience was not a significant factor.
\end{abstract}

Key Words:

Extramedullary Tibial Instrumentation, Total Knee Arthroplasty, Computer Navigation

\section{INTRODUCTION}

The success of a total knee arthroplasty (TKA) depends on the surgeon's ability to ensure proper extremity alignment and component position. Inaccurate implant position and mechanical misalignment can lead to accelerated implant wear and loosening. Studies have suggested that alignment errors of greater than $3^{\circ}$ are associated with more rapid failure and less satisfactory functional results ${ }^{1-3}$.

Mechanical alignment guides have improved the accuracy of implant alignment. However, errors in implant and limb alignment continue to occur. It has been estimated that errors in tibial and femoral alignment greater than $3^{\circ}$ occur in at least $10 \%$ of TKA cases. This is true, even with experienced surgeons using mechanical alignment systems of modern design ${ }^{1}$.

It is difficult to accurately determine the correct location of crucial alignment landmarks with standard instrumentation.
Even the most elaborate mechanical alignment systems rely on visual inspection to confirm the accuracy of the limb and implant alignment. Moreover, the design philosophies for mechanical alignment and sizing devices presume standardized bone geometry, but these standards may not apply to certain groups of patients, especially Asians. The tibial medullary canals of Asian patients are often bowed. $\mathrm{Ko}$, et $a l^{5}$, analysed a population of Chinese patients with varus arthritic knees. Results from the Ko study showed that use of the intra-medullary alignment guide resulted in an unacceptable tibial cut due to tibial bowing in $22 \%$ of cases. Computer-based alignment systems have been developed to address the limitations inherent in mechanical total knee instrumentation. However the adoption rate of these systems is not very high. Many surgeons show good long-term good to excellent results with traditional techniques, thus negating the need for newer techniques (e.g., computer navigation), which are more complicated, costly and time-consuming.

The objective of this study was to use an image-free computer-assisted navigation system to assess the accuracy of a currently available, conventional mechanical extramedullary tibial alignment system. This alignment system is based on predetermined and fixed anatomical landmarks, proximally and distally. In addition, we also set out to determine if surgeon's level of experience is a factor in successful use of the alignment system.

\section{MATERIALS AND METHODS}

The study period was from July 2009 to January 2010. Fifty consecutive patients comprised the sample for this study. All TKAs were performed at our institution by 2 surgeons, JS (author), and SS. JS' experience in TKA totalled less than one year, while SS had more than 5 years experience, with an average of 120 cases performed annually. All TKA implants were of the Stryker Scorpio ${ }^{\circledR}$ NGR Posterior Stabilized (PS) NRG design. All procedures were performed in conjunction with the Stryker ${ }^{\circledR}$ Navigation Computer-Assisted Surgery (CAS) System - eNact Knee (3.1) module, which is a nonimage computer navigation system.

Corresponding Author: Dr. Chee Eng Keong, Department of Orthopaedics and Traumatology, Joint Replacement Unit, Hospital Kuala Lumpur, 50586 Jalan Pahang, Kuala Lumpur, Malaysia Email: cheeengkeong@yahoo.com 


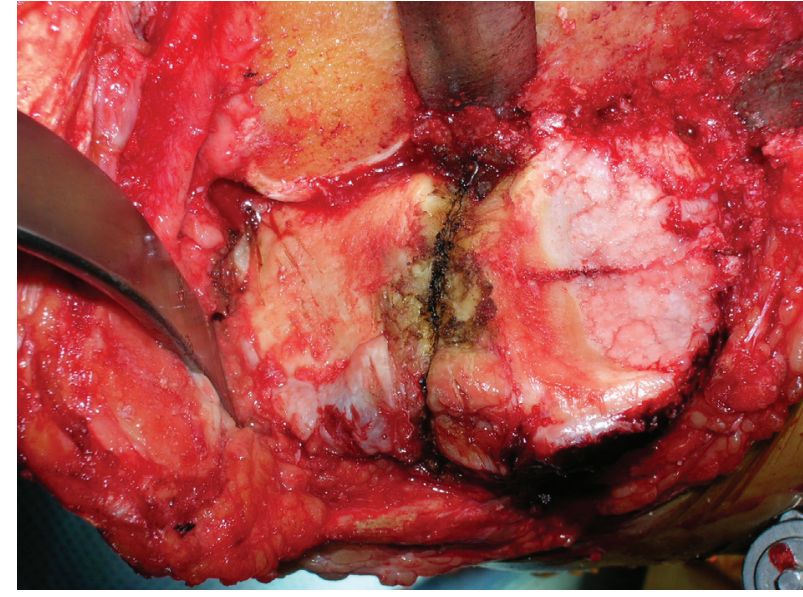

Fig. 1: Line drawn from insertion of the posterior cruciate ligament thru intercondylar eminence to the medial third of the tibial tubercle as landmark for proximal tibial extramedullary jig placement.

While the lower limb was being prepared and draped, special attention was taken to ensure that all anatomical landmarks over the ankle were clearly palpable. The TKAs were performed via the medial parapatellar approach. The patella was subluxated laterally. Retractors were placed posteriorly, medially and laterally to expose the entire tibial plateau. As for the CAS registration, the manufacturer's normal registration protocol was utilized. Tibial alignment was initially determined by using a currently available, Stryker extramedullary instrumentation alignment system, based on predetermined and fixed proximal and distal anatomical landmarks. Proximally, the jig was set by aligning the pins along a line drawn from the insertion of the posterior cruciate ligament, through the intercondylar eminence, to the medial third of the tibial tubercle (Fig 1). At the ankle, the rod was positioned using a spring device, with the distal end of the rod placed anterior to the tibial shin. Sagittal alignment was ensured by aligning the distal portion of the guide two fingerbreadths anterior to the crest of the distal tibia. As for the distal coronal alignment, we disregarded the possibility of tibial bowing or rotation, and no further adjustment was attempted. All readings were set at zero or neutral. The idea was to fix the proximal and distal points, and to form a line, connecting the two points. The zero-sloped tibial cutting block was positioned on the extramedullary alignment guide to allow a cut perpendicular to the axis of the tibia. The cutting jig was then pinned against the anterior surface of the proximal tibia to create neutral alignment in the coronal plane and $3^{\circ}$ in the sagittal plane. An outrigger, with a long perpendicular rod can be connected to the cutting jig for the surgeon to verify varus-valgus alignment.

Accuracy of the tibial extramedullary jig position was measured in the coronal and sagittal planes using an image-

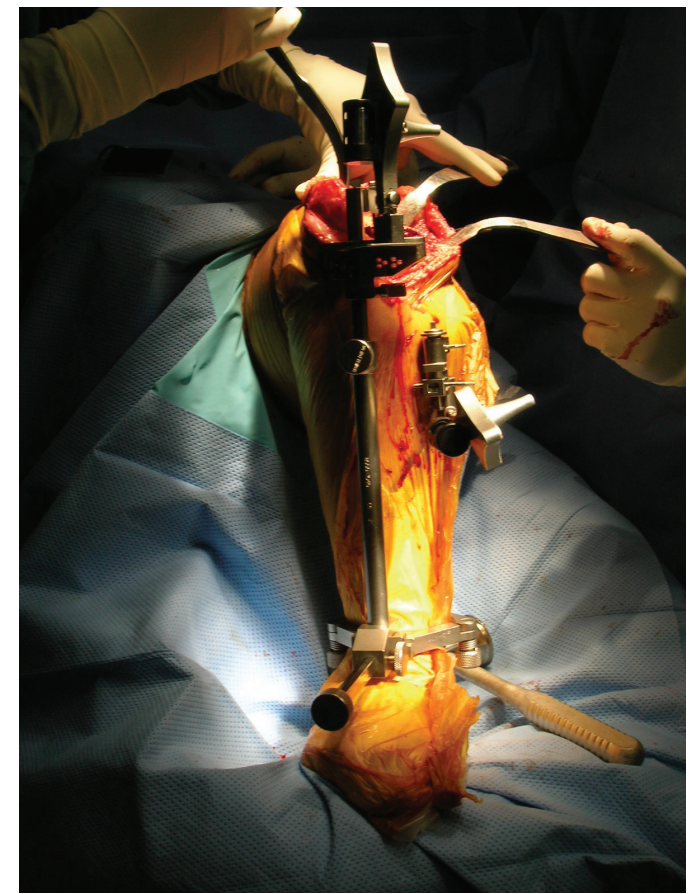

Fig. 2: Check plate to which attached a diode-containing tracker can be applied to the cutting blocks to determine their alignment.

free computer assisted navigation system with only one attempt. The navigation system has a check plate with an attached diode-containing tracker. This check plate can be applied to the cutting blocks that have been attached to the tibia to determine their alignment (Fig 2). Alignment readings were expressed in relation to the mechanical axis. Optimal results were considered to be a coronal axis of $0^{\circ}$ relative to the mechanical axis, and $3^{\circ}$ in the sagittal plane. Varus alignment was marked as a positive value, and valgus alignment was marked as a negative value.

SPSS (ver14) software for Windows was used to analyse the data. Nonparametric measurements were analyzed using the Mann Whitney ' $U$ ' test. A P value of 0.05 or less was considered significant.

\section{RESULTS}

Of the fifty patients included in this study, 39 were female, and 11 male. The mean age of the patients was 64 years old (SD range 46-81). All were diagnosed with primary osteoarthritis. There were 60 knees ( 10 bilateral; 6 single stage, sequential bilateral TKA, and 4 others bilateral TKA performed as separate surgeries). Thirty were right-sided, while the other 30 were left-sided knees.

Each surgeon performed thirty TKAs for this study, 15 right knees and 15 left knees. The mean patient age was $63 \mathrm{y}$ for 


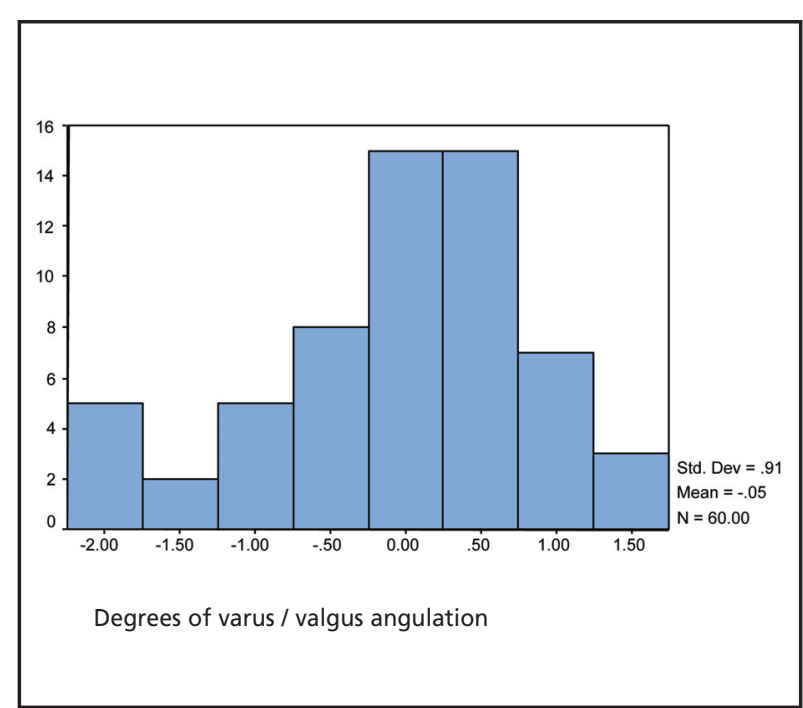

Fig. 3: Distribution of coronal alignment in degree versus number of patient.

SS' patients and 66 years for JS' patients. Four knees were excluded, three due to the placement failure of the extramedullary tibial jig, after application of the navigation tracker. The remaining excluded knee was a case of elephantiasis, with gross soft tissue envelope.

The 1 mean coronal angle achieved was $0.1^{\circ}$ valgus (SD 0.9, range -2.0 to 1.5 ), from the perpendicular to the mechanical axis. The SS group achieved a mean coronal angle of $0.3^{\circ}$ varus (SD 0.8, range -2.0 to 2.0), while the JS group achieved a mean coronal angle of $0.4^{\circ}$ valgus (SD 0.9 , range -2.0 to 1.5 ), each from the perpendicular to the mechanical axis.

If the 'acceptable' alignment is considered to be within $2^{\circ}$, or within $3^{\circ}$ of the perpendicular to the mechanical axis, 60 knees $(100 \%)$ had the tibial components in this acceptable position. However, if the acceptable alignment was taken as within $1^{\circ}$ of the perpendicular to the mechanical axis, the tibial components were in acceptable positions in 50 knees $(83.3 \%)$, with 7 knees in valgus (11.7\%), and 3 knees in varus $(5 \%)$. Both surgeons were able to achieve $100 \%$ of alignment (i.e., up to within $2^{\circ}$ of the perpendicular to the mechanical axis). If acceptable alignment was considered to be within $1^{\circ}$ of the perpendicular to the mechanical axis, the SS group had $87 \%$, while the JS group showed $80 \%$, of cases. a difference that is not statistically significant. There was also no statistical significance between both surgeons, when they each used the extramedullary tibial jig to achieve good tibial alignment angle. The distribution of the tibial alignment of the jig is depicted in Figures $3 \& 4$.

The overall mean posterior slope achieved was $3.4^{\circ}$ (SD 1.6, range 1.0 to 9.0) from the axis with the SS group achieving a mean posterior slope angle of $3.2^{\circ}$ (SD 1.5, range 1.0 to 9.0 ), and the JS group achieving a mean posterior slope angle

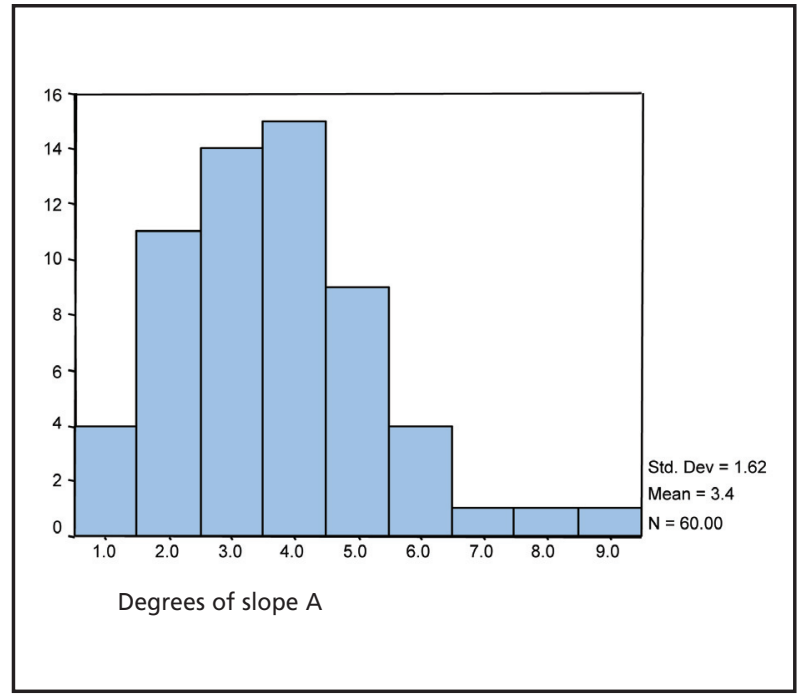

Fig. 4: Distribution of sagittal alignment in degree versus number of patient.

of $3.6^{\circ}$ (SD 1.7, range 1.0 to 8.0). If the acceptable alignment was defined as $3^{\circ}$ of posterior slope, the overall posterior slope alignment was acceptable in 16 knees (16.7\%). However, if acceptable alignment was defined as within $3-5^{\circ}$ of posterior slope, the alignment were in acceptable positions in 34 knees $(56.7 \%)$, with 7 knees $(11.7 \%)$ too posterior, and 19 knees (31.7\%) too anterior. When acceptable alignment was considered to be $3-5^{\circ}$ of posterior slope, the SS group had $63.3 \%$, and the JS group had only $50 \%$ of cases. Again, these differences were statistically not significant.

\section{DISCUSSION}

Traditionally, intra-medullary (IM) and extramedullary (EM) tibial alignment techniques are used to obtain neutral alignment during total knee arthroplasty. Several papers have reported that the extra and intramedullary systems are equally accurate in establishing tibial alignment ${ }^{2-4}$. However in the Asian population, the EM technique has proven to be more accurate by many authors, when compared to IM alignment. This is due to the higher incidence of tibia vara in this population ${ }^{2-4}$. Of note, the EM technique is less invasive, causes less bleeding, less embolisation of fat and marrow elements, and achieves reproducible mechanical alignment - even when there is an extra-articular deformity of the tibia.

Computer-navigated total knee arthroplasty have been shown to improve accuracy and reduce outliers, for implant placement ${ }^{2-4}$. Mikalho et al, analysed the difference in tibial alignment using intramedullary and extramedullary techniques, and compared these to the resulting tibial axis, as determined by a computer navigation system. The tibial alignment standard measure was determined by a spiral computed tomography (CT) study of each lower extremity. 
With very small differences between CT scan data, and navigation data for bony landmarks, it becomes evident that the navigation system data compares favourably to CT scan measures. Those authors therefore concluded that direct measurement of the mechanical axis using computer-guided surgical techniques for the tibia gives a greater degree of accuracy when compared to traditional alignment techniques. CT-scan analysis using the navigation system resulted in less than a $0.5^{\circ}$ difference from the navigation data in coronal alignment, and less than $0.3^{\circ}$ in sagittal alignment. Although the traditional alignment techniques results in measurements which may be within a few degrees, the addition of the possible errors in all planes may result in a much higher degree of error ${ }^{2}$.

It is an accepted fact that CAS is accurate, whereas the CT scan is more difficult and more costly. Thus, we decided to use CAS to analyse the accuracy of the extramedullary jig, and to assess the difference between two surgeons of different level of experience. The measurements were based on predetermined landmarks as described. We choose to use the EM guide, as we believe our group of patients have tibial bowing. This unique anatomical quality has been reported by other authors from this region. We also believe that to connect a line in a bowed or rotated tibia, one will need an intermediate fixed point, irrespective of the severity of the bowing or rotation. In the current study, adjustment of the guide based on the anterior tibial shin did not result in increased accuracy. This reflects the working basis of CAS meaning that registration of tibia mechanical alignment is only based on two points that connect the proximal and distal landmarks. Through the use of these fixed anatomical landmarks in placing the extramedullary tibial jig, we were able to achieve a high degree of accuracy in coronal alignment. The results also show that the accuracy in positioning the tibial cutting block was independent of the operator's experience as long as the predetermined anatomical landmarks are used as guides. The range of values obtained by trainees was marginally wider, as shown by Mahaluxmivala $\mathrm{J}$ et $a l^{3}$.

Our results also show that the use of extramedullary guide resulted in accurate coronal alignment (up to 1 degree varus/valgus), in up to $80-87 \%$ of patients. However the IM technique was not accurate for posterior slope or sagittal alignment. The posterior slope was more difficult to determine as it depends on the anterior tibial soft tissue envelope thickness. We estimate distances using the 2 fingerbreadth techniques. Thus, varying size of surgeons' fingers may be a significant factor in posterior slope accuracy. The adjustment at the distal tibial, which is located far from the cutting block, will also accentuate the error in taller patients.

The limitation of this study is that CAS has its own pitfalls in registration. If we do not perform the registration well, the accuracy of output will be affected, although this has not been verified in our study. Further, alignment obtained also does not necessarily reflect final implant position as error(s) can always occur during bone cut, due to flexing of the saw blade, sclerotic bone and differences in cementing technique.

\section{CONCLUSION}

The EM technique has been proven to be accurate in the Asian population, using predetermined fixed anatomical landmarks. This holds true for coronal, but not sagittal alignment. The accuracy of this technique was independent of operator experience. 


\section{REFERENCES}

1. Jeffery RS, Morris RW, Denham RA. Coronal alignment after total knee replacement. J Bone Joint Surg (Br). 1991; 73(5): 70914.

2. Ritter MA, Faris PM, Keating EM, Meding JB. Postoperative alignment of total knee replacement. Its effect on survival. Clin Orthop Relat Res. 1994; (299): 153-6.

3. Ritter MA, Herbst SA, Keating EM, Faris PM. Radiolucency at the bone-cement interface in total knee replacement. The effects of bone-surface preparation and cement technique. J Bone Joint Surg (Am). 1994; 76(1): 60-5.

4. Delp SL, Stulberg SD, Davies B, Picard F, Leitner F. Computer assisted knee replacement. Clin Orthop Relat Res. 1998 (354): 49-56.

5. Dennis DA, Channer M, Susman MH, Stringer EA. Intramedullary versus extramedullary tibial alignment systems in total knee arthroplasty. J Arthroplasty. 1993; 8(1): 43-7.

6. Brys DA, Lombardi AV, Jr., Mallory TH, Vaughn BK. A comparison of intramedullary and extramedullary alignment systems for tibial component placement in total knee arthroplasty. Clin Orthop Relat Res. 1991; (263): 175-9.

7. Maestro A, Harwin SF, Sandoval MG, Vaquero DH, Murcia A. Influence of intramedullary versus extramedullary alignment guides on final total knee arthroplasty component position: a radiographic analysis. J Arthroplasty. 1998; 13(5): 552-8.

8. Tang WM, Zhu YH, Chiu KY. Axial alignment of the lower extremity in Chinese adults. J Bone Joint Surg (Am). 2000; 82-A(11): 1603-8.

9. Matsuda S, Mizu-uchi H, Miura H, Nagamine R, Urabe K, Iwamoto Y. Tibial shaft axis does not always serve as a correct coronal landmark in total knee arthroplasty for varus knees. J Arthroplasty. 2003; 18(1): 56-62.

10. Chin PL, Yang KY, Yeo SJ, Lo NN. Randomized control trial comparing radiographic total knee arthroplasty implant placement using computer navigation versus conventional technique. J Arthroplasty. 2005; 20(5): 618-26.

11. Hernandez-Vaquero D, Suarez-Vazquez A, Sandoval-Garcia MA, Noriega-Fernandez A. Computer Assistance Increases Precision of Component Placement in Total Knee Arthroplasty with Articular Deformity. Clin Orthop Relat Res. $2009 ; 24$.

12. Mihalko WM, Krackow KA. Differences between extramedullary, intramedullary, and computer-aided surgery tibial alignment techniques for total knee arthroplasty. J Knee Surg. 2006; 19(1): 33-6.

13. Mahaluxmivala J, Bankes MJ, Nicolai P, Aldam CH, Allen PW. The effect of surgeon experience on component positioning in 673 Press Fit Condylar posterior cruciate-sacrificing total knee arthroplasties. J Arthroplasty. 2001; 16(5): 635-40. 